\title{
Renal function and cardiovascular events: relevance of eGFR and albuminuria in patients with diabetes
}

\author{
C. Delles • A. G. Jardine
}

Received: 8 July 2010 /Accepted: 1 September 2010 /Published online: 13 October 2010

(C) Springer-Verlag 2010

\begin{abstract}
Impaired renal function has been recognised as an independent cardiovascular risk factor in the general population and in patients with established cardiovascular disease. In this issue of Diabetologia, Drury et al. examined the association of two markers of renal function with cardiovascular outcome in patients with type 2 diabetes. They found that both estimated glomerular filtration rate (eGFR) and albuminuria were independent predictors, but that their incremental contribution to cardiovascular risk was modest compared with that of other risk factors. Both eGFR and albuminuria appear to integrate information from other risk factors and may be more suitable than populationbased risk scores for risk prediction in individuals, but further research is required to examine whether reduced eGFR and albuminuria mainly represent generalised vascular damage or if impaired renal function directly affects vascular structure and function in patients with type 2 diabetes.
\end{abstract}

Keywords Albuminuria $\cdot$ Cardiovascular risk · Glomerular filtration rate

$\begin{array}{ll}\text { Abbreviations } \\ \text { CKD } & \text { Chronic kidney disease } \\ \text { CVD } & \text { Cardiovascular disease } \\ \text { eGFR } & \text { Estimated glomerular filtration rate } \\ \text { FIELD } & \text { Fenofibrate Intervention and Event Lowering in } \\ & \text { Diabetes }\end{array}$

C. Delles $(\bowtie) \cdot$ A. G. Jardine

BHF Glasgow Cardiovascular Research Centre, Institute of Cardiovascular and Medical Sciences, University of Glasgow,

126 University Place,

Glasgow, Scotland G12 8TA, UK

e-mail: christian.delles@glasgow.ac.uk
JBS 2 Joint British Societies' guidelines on prevention of cardiovascular disease in clinical practice

MDRD Modification of Diet in Renal Disease

Cardiovascular disease (CVD) is the leading cause of death in patients with end-stage renal disease. Markers of cardiovascular damage, such as increased arterial stiffness and left ventricular hypertrophy, are commonly found in end-stage renal disease, where they predict cardiovascular outcome $[1,2]$. They are also evident in patients with less advanced chronic kidney disease (CKD) [3]. The link between renal impairment and CVD has been strengthened by the many studies demonstrating that subclinical renal impairment is common in the general population and in other groups without primary progressive renal disease and is an independent risk factor for death, cardiovascular events and hospitalisation [4]. Such studies have been facilitated by the development of estimates of renal function that do not require urine collection or infusion of tracer substances, based on the formula developed by Cockcroft and Gault in 1976 [5] to estimate creatinine clearance. At present, the equation most commonly used to calculate estimated glomerular filtration rate (eGFR) is the Modification of Diet in Renal Disease (MDRD) four-component formula [6]. This uses information on serum creatinine levels, age, sex and race, but does not require values for the patient's weight, blood urea nitrogen or albumin levels [7]. The formula undoubtedly has limitations: the correction for age may underestimate true renal function in the elderly, it is inaccurate in patients with a GFR $>60 \mathrm{ml} /$ $\mathrm{min}$, and the absence of a correction for weight may underestimate the true GFR at extremes of weight. However, despite these caveats it has become the basis for routine 
reporting of eGFR by biochemical laboratories in the UK and elsewhere.

A study in the present issue of Diabetologia by Drury et al. [8] examined the role of eGFR on cardiovascular events and mortality in 9,795 patients with type 2 diabetes but without advanced renal disease and found that a reduced eGFR was a risk factor for cardiovascular events. Despite being subject to the limitations discussed above, this study is important for a number of reasons. First, it is a large study with follow-up for up to 6 years, and the absence of a benefit of the primary intervention (fenofibrate vs placebo) on cardiovascular events [9] permits the inclusion of all patients in this post hoc analysis. Second, the authors have examined eGFR and urinary albumin excretion, observing that albuminuria increased cardiovascular risk independently of eGFR, and that there is an additive interaction between eGFR and protein excretion on cardiovascular outcomes. Third, the Fenofibrate Intervention and Event Lowering in Diabetes (FIELD) study recruited a low-risk population of patients with type 2 diabetes who were aged $50-80$ years and had near normal renal function as assessed by conventional methods (those with a serum creatinine of $>130 \mu \mathrm{mol} / 1$ were excluded and the mean value was only $79 \mu \mathrm{mol} / \mathrm{l}$ ); the 5 year mortality rate for this group was only $6.9 \%$. These data therefore give an insight into the impact of mild, sub-clinical renal dysfunction in the majority of patients with type 2 diabetes and have important implications [10].

What are the implications of the paper by Drury et al. [8] for clinical practice? Since the discovery of major cardiovascular risk factors in the Framingham study we have struggled to further refine our estimations of cardiovascular risk. Risk scores perform well on a population basis whereas a probability in per cent is not the most satisfactory clinical tool when it comes to treatment of individual patients. This is why markers that integrate several risk factors to indicate a patient's 'position' on the cardiovascular continuum [11] may help to estimate individual risk. In their current guidelines, the European Society of Hypertension and European Society of Cardiology acknowledge the potential benefit of intermediate cardiovascular phenotypes including endothelial function, vascular stiffness and carotid intima-media thickness to assess risk and to guide treatment decisions in patients with hypertension [12]. Similarly, in these and other guidelines (such as the Joint British Societies' guidelines on prevention of cardiovascular disease in clinical practice (JBS 2) [13]) it is recognised that CKD is a cardiovascular risk factor that should prompt physicians to target blood pressure and other modifiable risk factors more intensively. Assessment of renal function and proteinuria is thus part of the routine workup of patients with hypertension or diabetes $[12,14,15]$.

However, whilst the majority of risk scores (including JBS 2) label patients with CKD as a high-risk group who require intensive preventive therapy, it is surprising that a measure of renal function has not been incorporated in the currently available cardiovascular risk calculators, with the exception of some new scores such as QRISK2 [16]. Drury and colleagues offer a potential strategy for the subdivision of eGFR and proteinuria, with incremental, additive effects on outcomes. [9].

A criticism of the study is that (having excluded patients with a serum creatinine of $>130 \mu \mathrm{mol} / \mathrm{l})$ the main determinants of eGFR are age and sex. The authors highlight this issue and conclude that 'while their independent contributions to CVD risk (of eGFR and proteinuria) appear small when other risk factors are considered, because they capture risk related to a number of characteristics, they are excellent surrogate markers in clinical practice'. Moreover, they are readily measured and potentially remediable.

Why are eGFR and proteinuria markers of cardiovascular risk? The prevailing view is that impaired renal function and albuminuria are markers of general vascular damage that can be conveniently assessed by renal function. This may be the case for (micro)albuminuria, which is not always a specific marker for renal disease such as diabetic nephropathy. However, impaired renal function per se may cause direct cardiovascular effects. The nature of these effects is unclear in early stages of CKD that are not characterised by major changes in calcium and phosphate metabolisms or erythropoietin synthesis; mechanisms mediated by vascular calcification or anaemia thereby do not explain the findings. However, it is known that levels of some biomarkers (e.g. fibroblast growth factor 23) are elevated with minor loss of renal function, and that left ventricular hypertrophy may be present with even minor reductions in eGFR. Left ventricular hypertrophy might provide an explanation for the observation that reduced eGFR and albuminuria are associated with a dysproportionate increase in sudden death rather than nonfatal CHD events in the FIELD study [8]. These potential links require further exploration, and an alternative view is that renal dysfunction and proteinuria are manifestations of atheromatous vascular disease involving the renal vasculature. It is worth mentioning that novel markers of renal function, such as cystatin $C$ [17] and novel biomarkers of renal tubular damage (such as kidney injury molecule-1 and clusterin $[18,19])$, and urinary proteomic signatures $[20,21]$ may provide further insight into the relationship between $\mathrm{CKD}$ and cardiovascular risk.

Another interesting feature of the study [8] is that, in addition to baseline values, changes in renal function over a 2 year period also determined cardiovascular risk, and the authors speculate that improvement of renal function, for example, by appropriate medication, might improve cardiovascular outcome. Although there are reasons to believe this, one cannot draw definite conclusions from the reported observation, and changes in eGFR may be 'cosmetic', 
reflecting treatment with calcium channel blockers or reduced renin-angiotensin system blockade. However, the adverse effects of calcium channel blockers and the beneficial effects of angiotensin-converting enzyme inhibitors and angiotensin receptor blockers on glomerular pressure [22] may be more relevant for long-term renal (and potentially cardiovascular) outcome than any short-term effects on eGFR.

Overall, this study demonstrates the importance of minor reductions in renal function and the interaction with urinary albumin excretion on cardiovascular risk, even in low-risk individuals with type 2 diabetes. While the mechanisms and potential therapeutic implications of these observations will require further study, this is clear evidence that estimation of glomerular filtration rate even in patients with a normal serum creatinine level can improve cardiovascular risk stratification.

Duality of interest The authors declare that there is no duality of interest associated with this manuscript.

\section{References}

1. Zimmerli LU, Mark PB, Steedman T et al (2007) Vascular function in patients with end-stage renal disease and/or coronary artery disease: a cardiac magnetic resonance imaging study. Kidney Int 71:68-73

2. Pannier B, Guerin AP, Marchais SJ, Safar ME, London GM (2005) Stiffness of capacitive and conduit arteries: prognostic significance for end-stage renal disease patients. Hypertension 45:592-596

3. Briet M, Bozec E, Laurent S et al (2006) Arterial stiffness and enlargement in mild-to-moderate chronic kidney disease. Kidney Int 69:350-357

4. Go AS, Chertow GM, Fan D, McCulloch CE, Hsu CY (2004) Chronic kidney disease and the risks of death, cardiovascular events, and hospitalization. N Engl J Med 351:1296-1305

5. Cockcroft DW, Gault MH (1976) Prediction of creatinine clearance from serum creatinine. Nephron 16:31-41

6. Levey AS, Bosch JP, Lewis JB, Greene T, Rogers N, Roth D (1999) A more accurate method to estimate glomerular filtration rate from serum creatinine: a new prediction equation. Modification of Diet in Renal Disease Study Group. Ann Intern Med 130:461-470

7. Lin J, Knight EL, Hogan ML, Singh AK (2003) A comparison of prediction equations for estimating glomerular filtration rate in adults without kidney disease. J Am Soc Nephrol 14:2573-2580
8. Drury PL, Ting R, Zannino D et al (2010) Estimated glomerular filtration rate and albuminuria are independent predictors of cardiovascular events and mortality in type 2 diabetes mellitus: the Fenofibrate Intervention and Event Lowering in Diabetes (FIELD) study. Diabetologia. doi:10.1007/s00125-010-1854-1

9. Keech A, Simes RJ, Barter P et al (2005) Effects of long-term fenofibrate therapy on cardiovascular events in 9,795 people with type 2 diabetes mellitus (the FIELD study): randomised controlled trial. Lancet 366:1849-1861

10. Anavekar NS, McMurray JJ, Velazquez EJ et al (2004) Relation between renal dysfunction and cardiovascular outcomes after myocardial infarction. N Engl J Med 351:1285-1295

11. Dzau V, Braunwald E (1991) Resolved and unresolved issues in the prevention and treatment of coronary artery disease: a workshop consensus statement. Am Heart J 121:1244-1263

12. Mancia G, De Backer G, Dominiczak A et al (2007) 2007 Guidelines for the Management of Arterial Hypertension: The Task Force for the Management of Arterial Hypertension of the European Society of Hypertension (ESH) and of the European Society of Cardiology (ESC). J Hypertens 25:1105-1187

13. British Cardiac Society, British Hypertension Society, Diabetes UK, HEART UK, Primary Care Cardiovascular Society, The Stroke Association (2005) JBS 2: Joint British Societies' guidelines on prevention of cardiovascular disease in clinical practice. Heart 91(Suppl 5):v1-v52

14. Williams B, Poulter NR, Brown MJ et al (2004) Guidelines for management of hypertension: report of the fourth working party of the British Hypertension Society, 2004-BHS IV. J Hum Hypertens 18:139-185

15. American Diabetes Association (2010) Standards of medical care in diabetes - 2010. Diabetes Care 33(Suppl 1):S11-S61

16. Hippisley-Cox J, Coupland C, Vinogradova Y et al (2008) Predicting cardiovascular risk in England and Wales: prospective derivation and validation of QRISK2. BMJ 336:1475-1482

17. Shlipak MG, Sarnak MJ, Katz R et al (2005) Cystatin C and the risk of death and cardiovascular events among elderly persons. N Engl J Med 352:2049-2060

18. Vaidya VS, Ozer JS, Dieterle F et al (2010) Kidney injury molecule-1 outperforms traditional biomarkers of kidney injury in preclinical biomarker qualification studies. Nat Biotechnol 28: 478-485

19. Dieterle F, Perentes E, Cordier A et al (2010) Urinary clusterin, cystatin $\mathrm{C}$, beta2-microglobulin and total protein as markers to detect drug-induced kidney injury. Nat Biotechnol 28:463-469

20. Jantos-Siwy J, Schiffer E, Brand K et al (2009) Quantitative urinary proteome analysis for biomarker evaluation in chronic kidney disease. J Proteome Res 8:268-281

21. Mischak H, Rossing P (2010) Proteomic biomarkers in diabetic nephropathy - reality or future promise? Nephrol Dial Transplant 25:2843-2845

22. Delles C, Klingbeil AU, Schneider MP, Handrock R, Weidinger G, Schmieder RE (2003) Direct comparison of the effects of valsartan and amlodipine on renal hemodynamics in human essential hypertension. Am J Hypertens 16:1030-1035 